Research, Society and Development, v. 9, n. 11, e1729119519, 2020

(CC BY 4.0) | ISSN 2525-3409 | DOI: http://dx.doi.org/10.33448/rsd-v9i11.9519

Aplicação de filtros passa-baixa em modelos digitais de elevação para avaliação da diferença altimétrica entre os Pantanais do Negro e Nhecolândia

Application of low-pass filters in digital elevation models for evaluation of the altimetric difference between Pantanais do Negro e Nhecolândia

Aplicación de filtros de paso bajo en modelos de elevación digital para evaluar la diferencia altimétrica entre los Pantanais do Negro y Nhecolândia

Recebido: 22/10/2020 | Revisado: 27/10/2020 | Aceito: 05/11/2020 | Publicado: 08/11/2020

\title{
Alesandro Copatti
}

ORCID: https://orcid.org/0000-0001-8509-471X Universidade Federal de Mato Grosso do Sul, Brasil

E-mail: acopatti@hotmail.com

Deborah Mendes

ORCID: https://orcid.org/0000-0003-1804-192X

Serviço Geológico do Brasil, Brasil

E-mail: mendesdh@gmail.com

Ana Paula Garcia Oliveira

ORCID: https://orcid.org/0000-0001-8942-0703

Universidade Anhanguera, Brasil

E-mail: apg.bio@gmail.com

Fabrício Bau Dalmas

ORCID: https://orcid.org/0000-0001-7547-6642

Universidade Guarulhos, Brasil

E-mail: fdalmas@prof.ung.br

Antonio Conceição Paranhos Filho

ORCID: https://orcid.org/0000-0002-9838-5337

Universidade Federal de Mato Grosso do Sul, Brasil

E-mail: antonio.paranhos@pq.cnpq.br

\section{Resumo}

Os dados do radar interferométrico de abertura sintética SRTM apresentam grande potencialidade para estudos que envolvam a altimetria, uma vez que, além de estarem 
disponíveis gratuitamente na internet, também estão geocodificados, facilitando seu tratamento em Sistema de informações geográficas. Entretanto os dados SRTM apresentam algumas limitações de uso, entre elas o Efeito Dossel, consequência do modelo considerar o topo da vegetação como feição de relevo. Outra limitação, não menos importante, especialmente para a utilização desses dados em áreas planas, refere-se à precisão vertical desses dados, já que na área deste estudo, situada no Pantanal, uma diferença de apenas um metro tem um significado importante. O presente trabalho possui os objetivos de avaliar os dados de altimetria oriundos dos dados de SRTM, TOPODATA e do GLS; analisar a acurácia vertical, através da comparação dos dados destes sensores com os dados de altitude dos marcos geodésicos do Instituto Brasileiro de Geografia e Estatística; e aplicar Filtro PassaBaixa de Média Móvel para a correção do "Efeito Dossel" dos dados SRTM. A área de estudo escolhida para realizar todas essas análises está englobada entre os Pantanais do Negro e Nhecolância. A metodologia aplicada foi desenvolvida em ambiente SIG de plataformas gratuitas: GRASS-GIS, Quantum GIS e SPRING. Os dados SRTM utilizados permitiram avaliar a diferença altimétrica entre o Pantanal do Negro e a Nhecolândia. Já os filtros aplicados sobre os dados SRTM diminuíram substancialmente as principais feições associadas ao efeito dossel da vegetação.

Palavras-chave: Geotecnologias livres; Unidades de conservação da natureza; Altimetria.

\begin{abstract}
The SRTM synthetic aperture interferometric radar data have great potential for studies involving altimetry, since, in addition to being freely available on the internet, they are also geocoded, facilitating their treatment in Geographic Information System. However, the SRTM data has some usage limitations, including the Canopy Effect, a consequence of the model considering the top of the vegetation as a relief feature. Another limitation, no less important, especially for the use of these data in flat areas, refers to the vertical precision of these data, since in the area of this study, located in the Pantanal, a difference of only one meter has an important significance. The present work aims to evaluate the altimetry data from SRTM, TOPODATA and GLS data; to analyze the vertical accuracy, by comparing the data of these sensors with the altitude data of the geodesic landmarks of the Brazilian Institute of Geography and Statistics; and apply Moving Average Low Pass Filter to correct the "Canopy Effect" of SRTM data. The study area chosen to carry out all these analyzes is included between the Pantanais do Negro and Nhecolância. The applied methodology was developed in a free platform GIS environment: GRASS-GIS, Quantum GIS and SPRING.
\end{abstract}


The SRTM data used allowed us to assess the altimetric difference between the Pantanal do Negro and Nhecolândia. Filters applied to SRTM data substantially reduced the main features associated with the canopy effect of vegetation.

Keywords: Free geotechnology; Units for conservation of nature; Altimetry.

\section{Resumen}

Los datos del radar interferométrico de apertura sintética SRTM tienen un gran potencial para estudios de altimetría, ya que además de estar disponibles gratuitamente en Internet, también están geocodificados, facilitando su tratamiento en el Sistema de Información Geográfica. Sin embargo, los datos de SRTM tienen algunas limitaciones de uso, incluido el efecto dosel, una consecuencia de que el modelo considera la parte superior de la vegetación como una característica de relieve. Otra limitación, no menos importante, especialmente para el uso de estos datos en áreas planas, se refiere a la precisión vertical de estos datos, ya que en el área de este estudio, ubicada en el Pantanal, una diferencia de solo un metro tiene un significado importante. El presente trabajo tiene como objetivo evaluar los datos altimétricos de SRTM, TOPODATA y GLS; analizar la precisión vertical, comparando los datos de estos sensores con los datos de altitud de los hitos geodésicos del Instituto Brasileño de Geografía y Estadística; y aplique el filtro de paso bajo de media móvil para corregir el "efecto de dosel" de los datos SRTM. El área de estudio elegida para realizar todos estos análisis se incluye entre Pantanais do Negro y Nhecolância. La metodología aplicada se desarrolló en un entorno GIS de plataforma libre: GRASS-GIS, Quantum GIS y SPRING. Los datos de SRTM utilizados nos permitieron evaluar la diferencia altimétrica entre el Pantanal do Negro y Nhecolândia. Los filtros aplicados a los datos de SRTM redujeron sustancialmente las principales características asociadas con el efecto de dosel de la vegetación.

Palabras clave: Geotecnologías libres; Unidades de conservación de la naturaleza; Altimetría.

\section{Introdução}

O Pantanal é uma das maiores áreas alagáveis do mundo, com aproximadamente 147.000 km2, abrigando sítios de relevante importância pela Convenção de Áreas Úmidas e foi declarado Patrimônio Nacional pela Constituição Federal (Brasil, 1988). Em função da baixa declividade e a quase completa ausência de elevações fisiográficas na planície, a vegetação arbórea do Pantanal é encontrada em locais mais elevados, entre um a dois metros 
acima da vegetação de gramíneas, sobre as chamadas "cordilheiras".

Os dados do radar interferométrico de abertura sintética SRTM apresentam grande potencialidade para estudos que envolvam a altimetria ou produtos derivados. Estes dados estão disponíveis gratuitamente na internet e também estão geocodificados (Zyl, 2001), facilitando seu tratamento em Sistema de Informações Geográficas (SIG). Far et al. (2007) informaram que, para o continente sul-americano, o erro absoluto de altimetria é de 6,2 metros, o erro relativo de altimetria é de 5,5m e o erro absoluto de geolocalização é de 9,0m. Dados que foram confirmados por Rodrigues et al. (2006).

Hyga et al. (2006), avaliando a precisão vertical dos dados SRTM no Estado do Mato Grosso do Sul (MS) e confrontando as altitudes do SRTM com as altitudes ortométricas dos marcos da Rede GPS (Global Positioning System) daquele Estado, concluíram que o SRTM atende ao Padrão de Exatidão Cartográfica PEC) até a escala de 1:30.000 (Classe A) ou 1: 20.000 (Classe C). Entretanto, a utilização do SRTM apresenta algumas limitações. Uma delas, particularmente de grande expressão na área de estudo do presente artigo, é o "efeito dossel", devido às características dos sensores de radar utilizados registrarem como superfície do terreno o topo das estruturas encontradas sobre o modelado terrestre, sejam elas edificações ou o dossel da vegetação. Outra limitação, não menos importante para a utilização desses dados em áreas muito planas, refere-se à precisão vertical desses dados (Valeriano \& Abdon, 2007).

Diante do exposto, os objetivos deste artigo foram: avaliar os dados de altimetria oriundos dos dados altimétricos da SRTM (Jarvis, 2008), TOPODATA (INPE, 2011) e do GLS (Global Land Survey), quanto à acurácia vertical, comparando-os com os dados de altitude dos marcos geodésicos do Instituto Brasileiro de Geografia e Estatística (IBGE); e uma análise da aplicação de Filtro Passa-Baixa de Média Móvel para a correção do "Efeito Dossel" dos dados SRTM.

\section{Metodologia}

A Metodologia de Pesquisa aplicada no presente trabalho foi o Estudo de Caso, de modo que os aspectos de altimetria da área de estudo, Pantanal do Negro e Nhecolândia, foram analisados através de dados de diferentes sensores. Dessa maneira, realizou-se uma primeira análise qualitativa, para se definir quais sensores seriam utilizados, e que se expandiu para investigações quantitativas, ao se comparar os resultados dos sensores. Esse método de conduzir a pesquisa segue o que é descrito por Yin (2015, apud Pereira et. al, 
Research, Society and Development, v. 9, n. 11, e1729119519, 2020

(CC BY 4.0) | ISSN 2525-3409 | DOI: http://dx.doi.org/10.33448/rsd-v9i11.9519

2018), o qual afirma que os estudos quantitativos e os qualitativos, aplicados de maneira complementar, podem fornecer um entendimento mais completo do fenômeno em estudo.

\section{1 Área de estudo}

A área de estudos compreende a porção dos Pantanais da Nhecolândia, do Abobral e do Miranda contida nos limites geográficos do Parque Estadual do Pantanal do Rio Negro (PEPRN) e sua Zona de amortecimento, para avaliação da potencialidade de utilização dos dados SRTM na análise de feições fisiográficas do Pantanal (Figura 1). O Parque Estadual do Pantanal do Rio Negro (PEPRN) é a única Unidade de Conservação da Natureza (UC) de Proteção Integral do Pantanal, em Mato Grosso do Sul, integra as áreas núcleo da Reserva da Biosfera do Pantanal (IMASUL, 2008).

Figura 1. Localização da área de estudo, Parque Estadual do Pantanal do Rio Negro e sua Zona de Amortecimento.

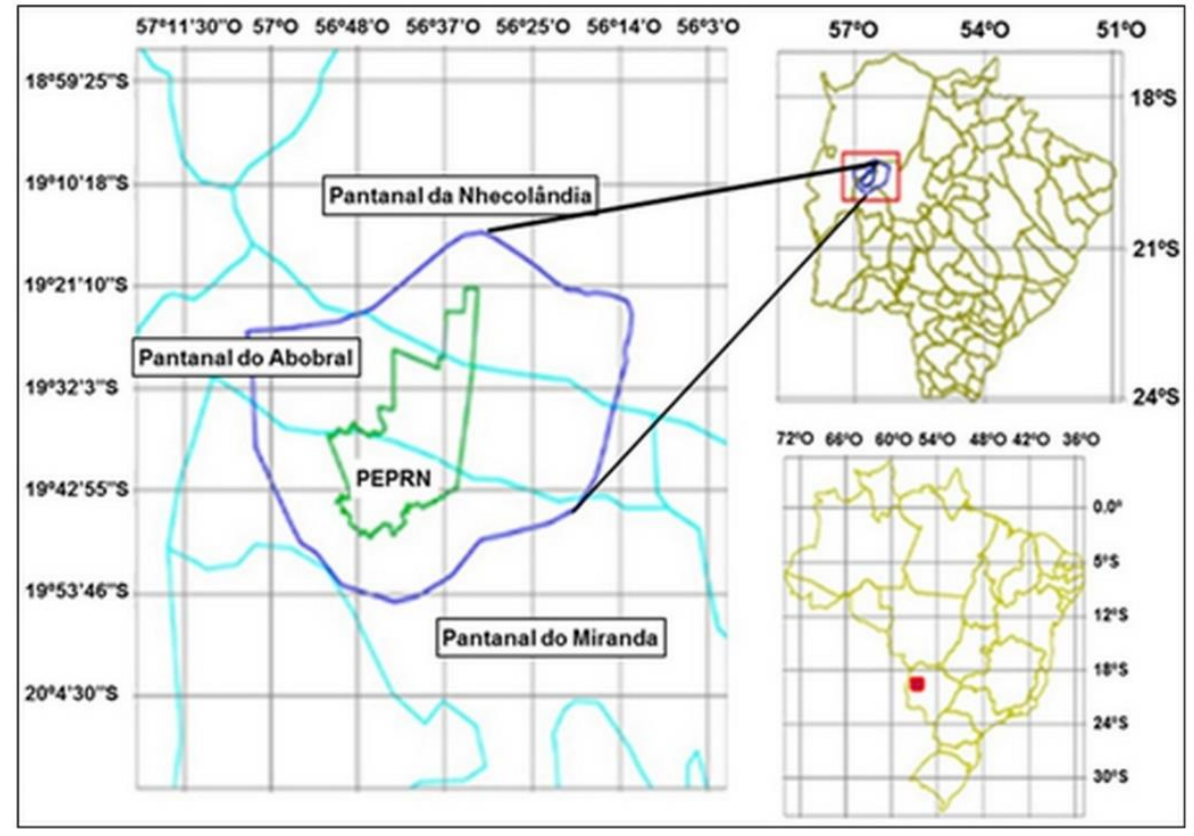

Fonte: Autores.

\subsection{Avaliação comparativa dos Modelos Digitais de Elevação}

Os dados de altitudes geométricas da Rede Geodésica Brasileira de GPS foram adquiridos do Banco de Dados Geodésicos do IBGE (IBGE, 2010). Os pontos contendo as altitudes geométricas foram agrupados em um arquivo único (no formato *.csv), que foi 
importado como amostras de elevação em um modelo numérico do programa SPRING (INPE, 2006), permitindo a sobreposição desses dados com os demais produtos avaliados.

Os dados de altimetria do Banco de Dados Geomorfométricos do Brasil TOPODATA (INPE, 2011) foram exportados do portal do Instituto Nacional de Pesquisas Espaciais para o banco de dados do SPRING (INPE, 2006). Para este mesmo banco foram importados os dados SRTM - versão 4.1 (Jarvis, 2008) e dados TOPODATA e GLS 2005. A próxima etapa foi coleta dos valores de altimetria desses três produtos, coincidente com os locais onde há dados pontuais da Rede Geodésica Brasileira.

\subsection{Filtragem dos dados SRTM: contribuição à utilização do SRTM em áreas de baixo gradiente topográfico}

No software GRASS (GRASS Development Team, 2002), o SRTM foi filtrado com um filtro de passa-baixa ("r.neighbors") utilizando a operação de média, com a janela (quadrada) do filtro variando de $3 \mathrm{X} 3,7 \mathrm{X} 7,11 \mathrm{X} 11$ e $25 \mathrm{X} 25$ pixels. Esses resultados foram exportados para o formato GEOTIFF e novamente importados para o SPRING, onde foram geradas as curvas de nível com equidistância de um metro. Ainda no GRASS, também foram gerados histogramas de frequência para 3 regiões distintas envolvendo a Zona de Amortecimento do Parque, aproximadamente orientadas de NNE para SSO, a fim de comparar graficamente os resultados.

O histograma de frequências foi utilizado como parâmetro para a avaliação preliminar das filtragens utilizadas. Após a determinação empírica do filtro mais adequado aos objetivos deste trabalho foi realizada a derivação das variáveis "bacias" e "segmentos de fluxo". Essas

variáveis matriciais foram convertidas para o formato vetorial e essas linhas foram sobrepostas à imagem do sensor TM (Landsat 5) de 2011, para verificação da consistência geral dos resultados.

\section{Resultados e Discussão}

\subsection{Avaliação comparativa dos Modelos Digitais de Elevação}

A Figura 2 apresenta a distribuição espacial dos marcos geodésicos do IBGE sobre o DEM GLS2005. 
Figura 2. Marcos geodésicos do IBGE sobre o DEM GLS 2005, órbita 225 e ponto 074.

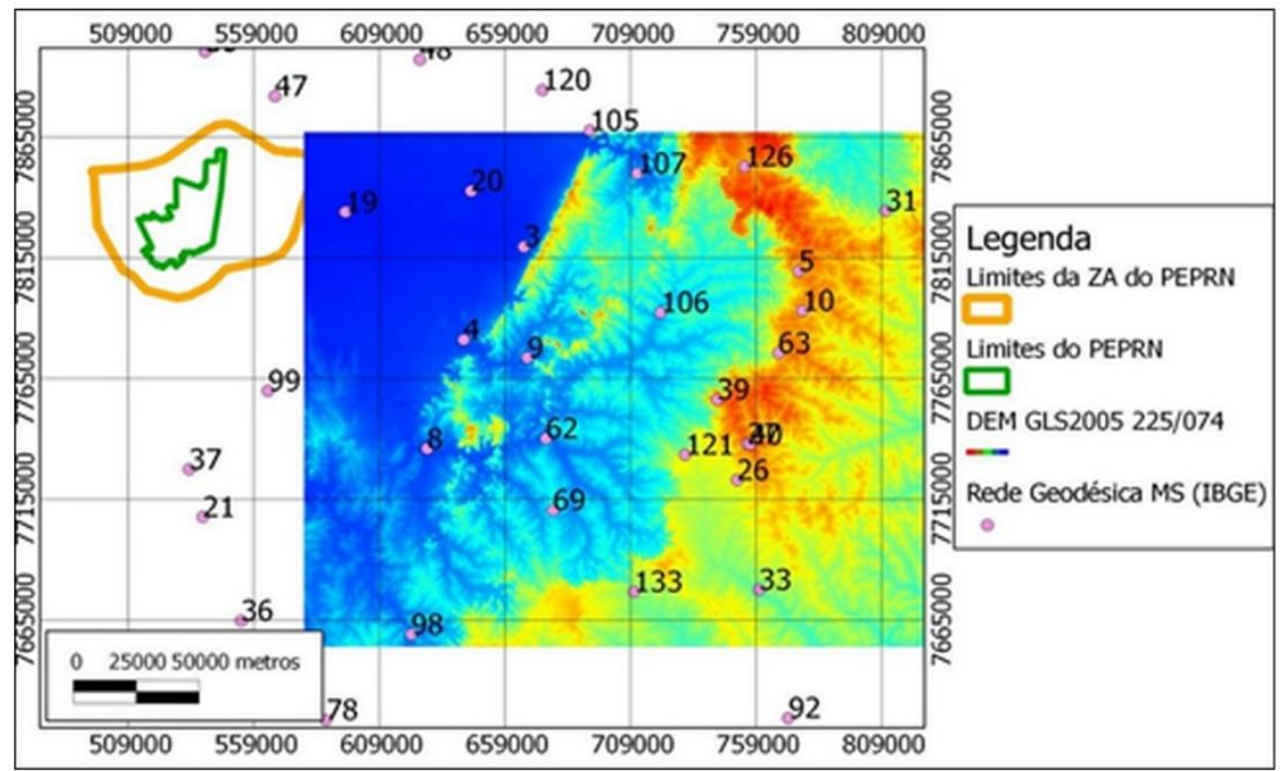

Fonte: Autores.

A Tabela 1 apresenta os valores referentes às diferenças máximas, média e desviopadrão, quando comparados os dados provenientes do SRTM, TOPODATA e Modelo Digital de Elevação com dados dos Marcos Geodésicos do IBGE. A partir destas análises comparativas, percebe-se que os dados do TOPODATA um pouco melhores do que os dados SRTM, embora tenha sido observado que há um número expressivo de dados do TOPODATA onde os erros são maiores do que o SRTM.

Tabela 1. Análise comparativa entre a posição dos Marcos Geodésicos e os respectivos registros nos dados de SRTM, TOPODATA e MDE.

\begin{tabular}{lccc}
\hline \multirow{2}{*}{ COMPARATIVO } & \multicolumn{3}{c}{ DIFERENÇA $(\mathrm{m})$} \\
& MÁXIMA & MÉDIA & DESVIO PADRÃO \\
\hline IBGE e SRTM & 13,3 & 6,93 & 3,32 \\
IBGE e TOPODATA & 12,2 & 6,66 & 3,16 \\
Marcos Geodésicos e MDE & 5,8 & 2,33 & 1,50 \\
\hline
\end{tabular}

Fonte: Autores. 
Research, Society and Development, v. 9, n. 11, e1729119519, 2020

(CC BY 4.0) | ISSN 2525-3409 | DOI: http://dx.doi.org/10.33448/rsd-v9i11.9519

A avaliação das diferenças entre Marcos Geodésicos e MDE (Tabela 1) apresentou os melhores resultados, com a maior diferença encontrada de aproximadamente 5,8 metros. Portanto, o MDE pode ser considerado melhor que o SRTM e que o TOPODATA, para a região avaliada. Dos 23 pontos comparados (marcos geodésicos), observou-se que em 10 deles as diferenças IBGE-TOPODATA foram maiores que as diferenças IBGE-SRTM, indicando que a interpolação realizada nos dados TOPODATA também produziu diminuição da acurácia. Esse pode ser um dos motivos das variáveis morfométricas serem ressaltadas quando extraídas do produto TOPODATA, como, por exemplo, as declividades acima de 45 graus.

Como as diferenças entre os dados do IBGE e GLS2005 apresentaram valores positivos e negativos (erros aleatórios), esses arquivos foram convertidos para números absolutos para a avaliação quantitativa dessas diferenças. Assim, foi possível observar que essas diferenças são quase sempre bem menores do que as observadas para o SRTM e para o TOPODATA.

Já as diferenças nas comparações entre IBGE e SRTM e entre IBGE e TOPODATA apresentaram sempre valores positivos, mostrando que os dados SRTM e TOPODATA estão sempre subestimados em relação aos marcos geodésicos do IBGE (erro sistemático), na região avaliada. Esses dados são consistentes com os obtidos por Ludwig e Schneider (2006), que avaliaram o MDT (Modelo Digital de Terreno) extraído do SRTM na banda X. O MDT foi avaliado quanto ao aspecto, à declividade, ao ângulo de incidência local e uso do solo e os autores concluíram que as altitudes são superestimadas nos relevos movimentados e subestimadas nos relevos planos.

\subsection{Correção dos dados SRTM: utilização de Filtro Passa-Baixa de Média Móvel Para a} Correção do "Efeito Dossel"

A Figura 3 evidencia a redução da interferência do dossel da vegetação e a elevação da altimetria do fundo dos corpos de água na geração das curvas de nível, após a aplicação do filtro com janela de 25x25, preservando o padrão regional de elevação do terreno. Nota-se, aproximadamente no centro desta figura, uma isolinha aproximadamente retangular, que ocorre devido ao processamento do filtro e, principalmente, porque os dados estão em números inteiros e não foram convertidos para ponto flutuante. 
Figura 3. Isolinhas desenhadas sobre o SRTM, após a aplicação do filtro de média de vizinhança com a janela de $25 \times 25$ pixels, sobre a imagem TM (Landsat 5), órbita/ponto 226/073, de 22 de abril de 2011, composição: RGB 453.

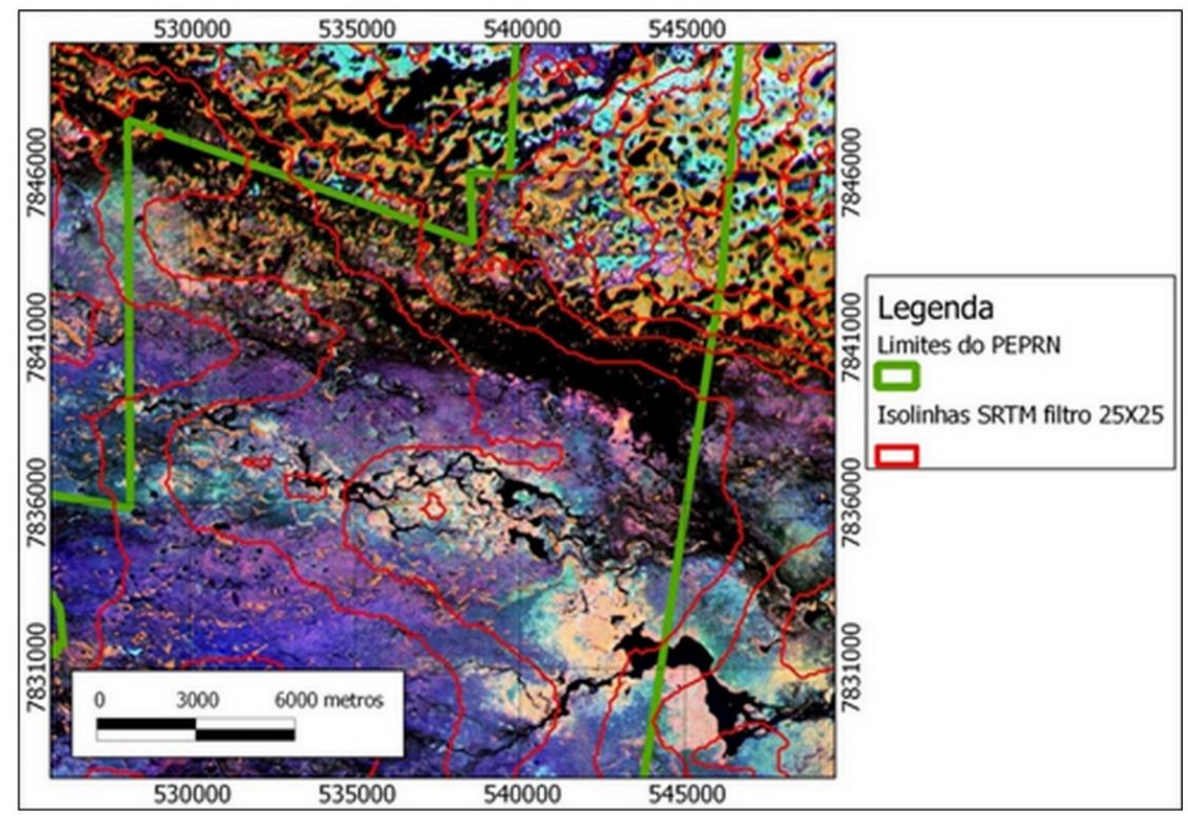

Fonte: Autores.

A Figura 4 apresenta o mapa hipsométrico da área de estudo, elaborado sobre o SRTM original, e a Figura 5 apresenta o Mapa Hipsométrico elaborado sobre o SRTM após a aplicação do filtro de $25 \times 25$.

Figura 4. Mapa Hipsométrico da área de estudo, elaborado sobre o SRTM original.

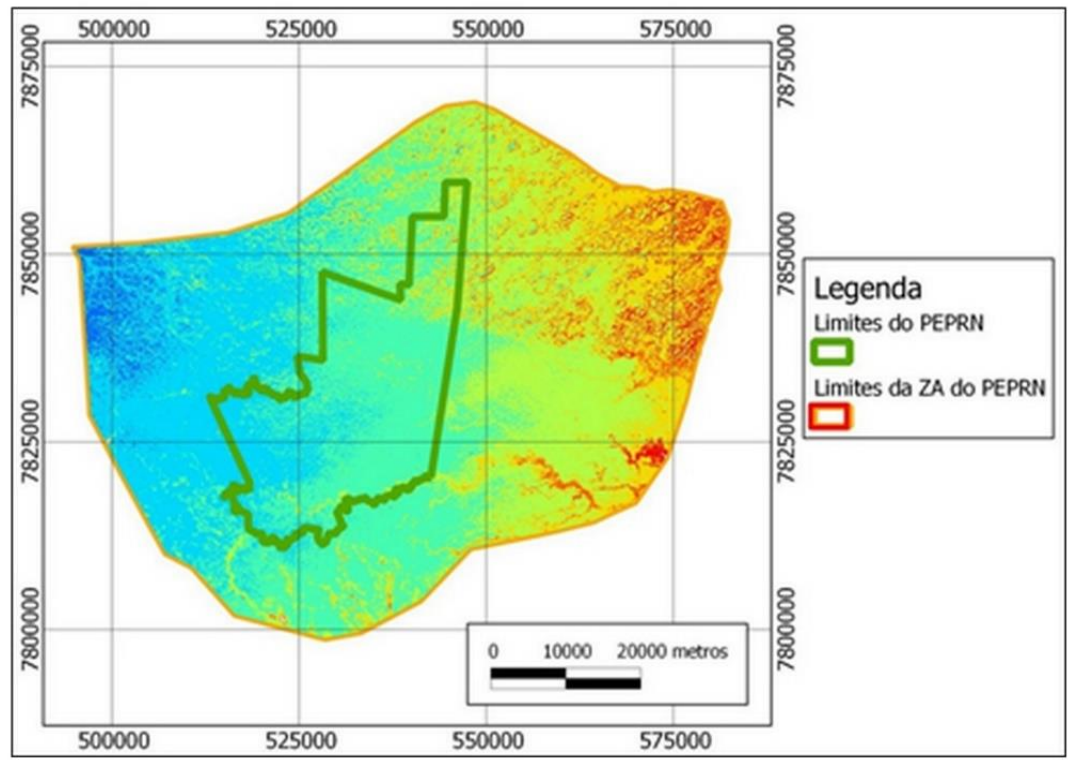

Fonte: Autores. 
Figura 5. Mapa Hipsométrico da área de estudo, elaborado sobre o SRTM após a aplicação do filtro de $25 \times 25$.

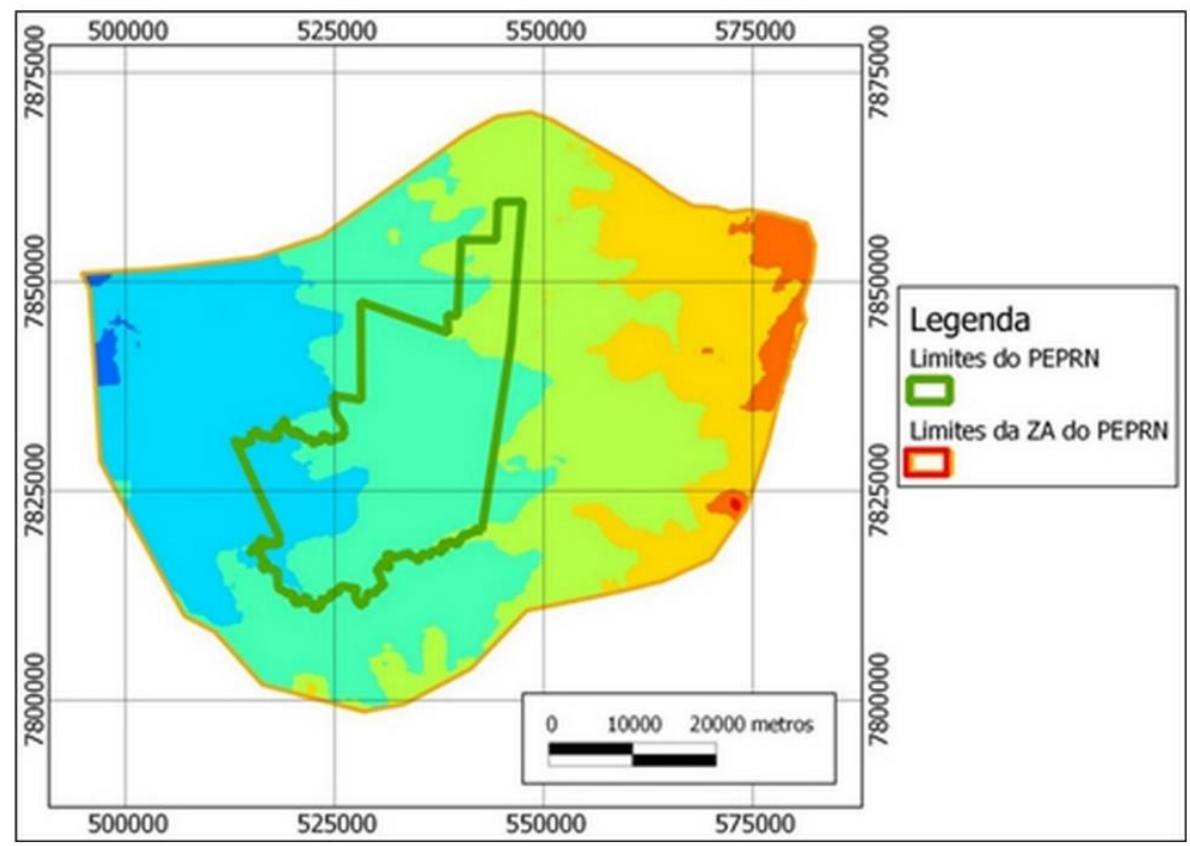

Fonte: Autores.

A aplicação de filtro de média do tipo passa-baixa com o tamanho da janela de $25 \times 25$ foi eficiente para suavizar as feições extremas (dossel de vegetação e fundos das lagoas e corpos de água) mantendo o padrão regional de declividade do terreno. Dessa forma, a partir do DEM resultante desse processo foi possível identificar as diferenças no relevo, particularmente entre o Pantanal da Nhecolândia e o Pantanal do Abrobral, marcada pelos brejos do Abobral ao sul e pelas Lagoas da Nhecolândia ao norte. Essa diferença pode ser observada tanto nos perfis do modelo de elevação quanto nas curvas de nível traçadas de metro em metro. Semelhante resultado tiveram Marini et al. (2017) onde a representação do relevo da Nhecolândia a partir do uso de MDT possibilitou identificar pequenas variações de altitude do relevo, permitindo analisar regiões de lagoas e vazantes na Nhecolândia, que são os principais elementos da paisagem da região em estudo, onde ele ressalta o efeito dossel detectados pelo uso de MDT.

\subsection{Avaliação da derivação de variáveis morfométricas após a aplicação do filtro de passa-baixa de média}

A Figura 6 apresenta as derivações dos segmentos de fluxo gerados a partir do SRTM sem tratamento e a partir da utilização do filtro utilizado. A escolha desse tamanho de janela 
também foi em função do tamanho e padrão das formações arbóreas que maximizam esse efeito, ou seja, as formações florestais típicas dessa região conhecidas como cordilheiras (em tons alaranjados na imagem ao fundo).

Figura 6. Segmentos de fluxo gerados a partir do SRTM e a partir do filtro com janela de 25x25. Ao fundo, imagem Landsat de 2011, composição: vermelho=b4; verde=b5 e azul=b3.

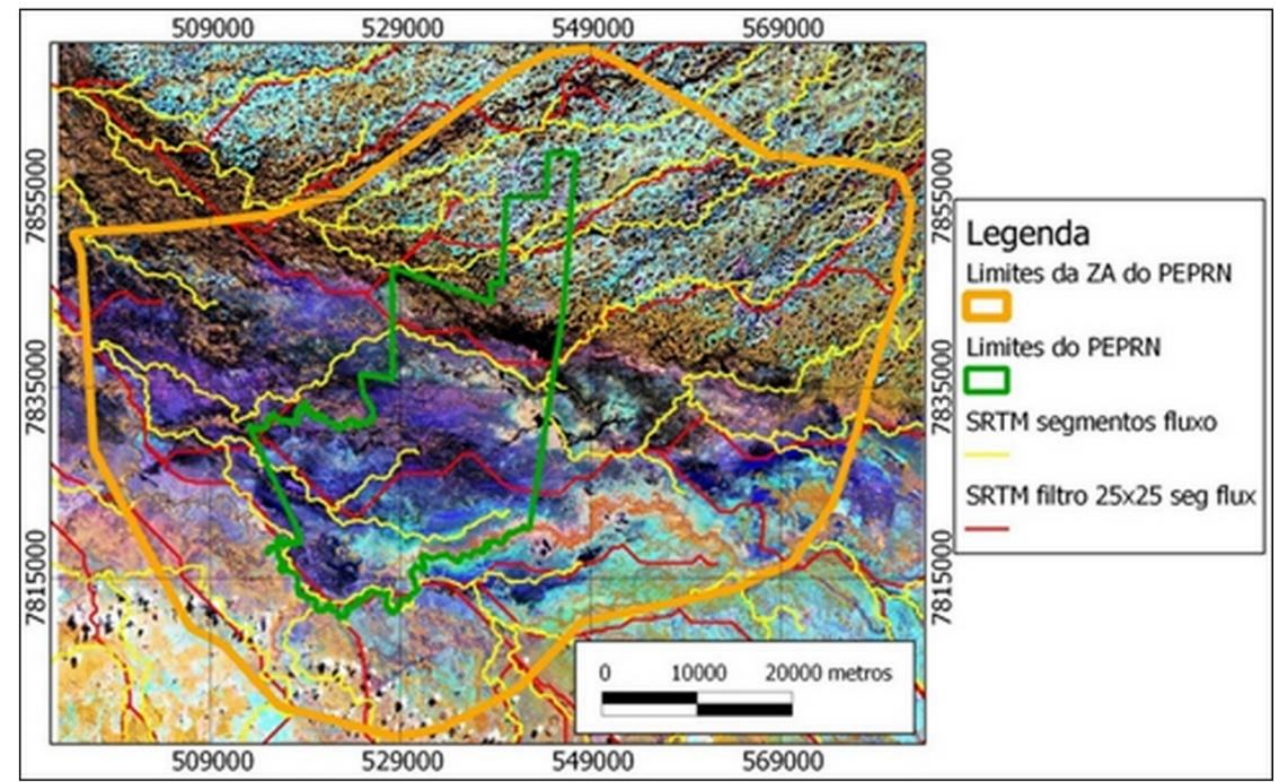

Fonte: Autores.

A Figura 7 apresenta os limites das Bacias Hidrográficas geradas a partir do SRTM sem tratamento e as geradas a partir da aplicação do filtro de passa-baixa com janela quadrada de média de $25 \times 25$. Observa-se que na região do Abobral, os segmentos de fluxo e os divisores das bacias hidrográficas apresentaram um padrão aproximadamente retilíneo, indicando a produção de artefatos, o que é previsível devido à declividade extremamente baixa aliado ao fato de os dados do SRTM estarem em números inteiros.

Ou seja, são consideradas altitudes apenas de metro em metro, porém as diferenças reais aí encontradas são inferiores a esses valores. Observando-se a Figura 7, nota-se maior nitidez no padrão de declividade, enquanto a Figura 6 apresenta grande dificuldade para observar esse padrão devido, principalmente, ao efeito dossel, conforme já comentado. 
Figura 7. Limites das bacias hidrográficas gerados a partir do SRTM e a partir do filtro com janela de 25x25. Ao fundo, imagem Landsat de 2011, composição: vermelho=b4; verde=b5 e azul=b3.

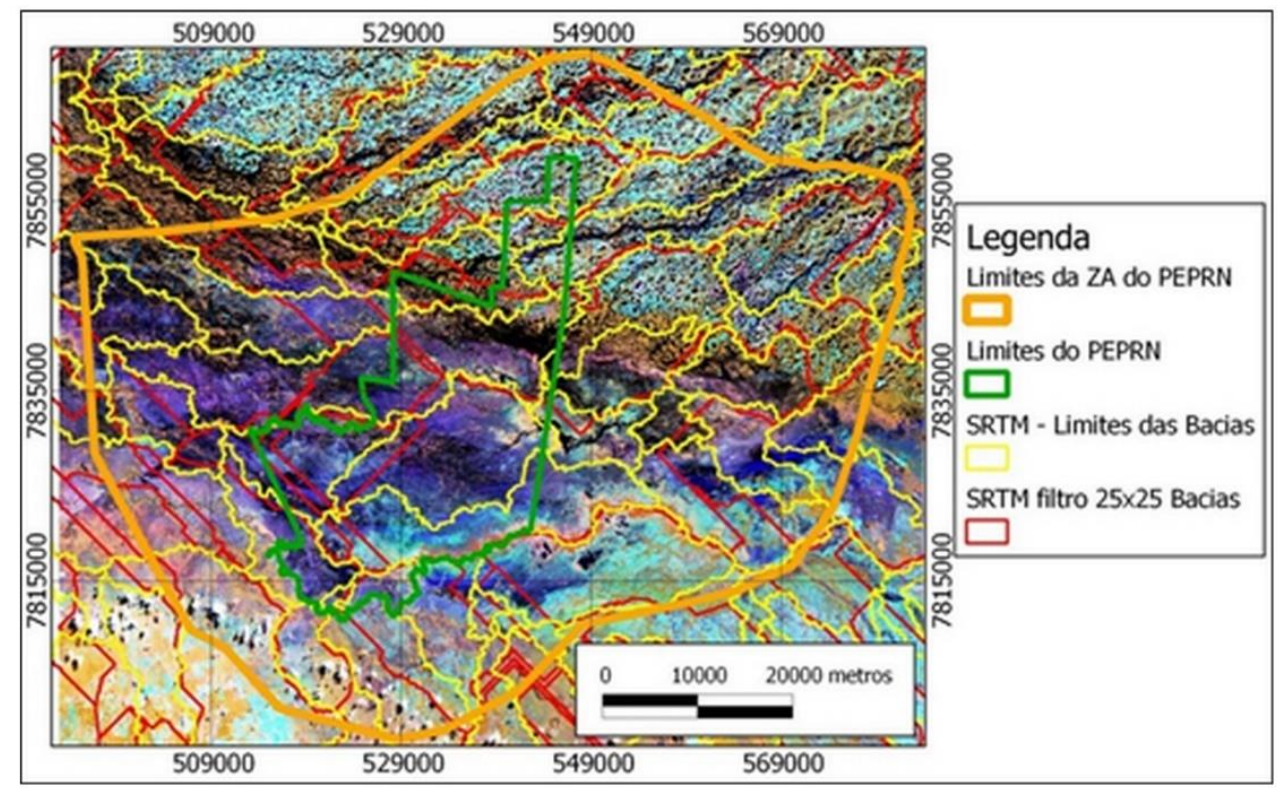

Fonte: Autores.

\section{Considerações Finais}

Em relação às análises realizadas, notou-se uma limitação referente à precisão vertical dos dados SRTM em áreas muito planas, já que na área de estudo uma diferença de apenas um metro tem um significado importante. Disso decorre que, ao se derivarem informações morfométricas (baixa declividade aliado a presença de um mosaico de formações florestais intercaladas com massas de água e áreas de pastagem nativa) a partir desses dados em áreas da Planície Pantaneira, ocorrem "artefatos", ou seja, a criação de feições derivadas que não representam a realidade. Deve-se observar que todos os Marcos Geodésicos estão localizado em "áreas limpas", ou seja, sem vegetação arbórea.

Para a derivação de variáveis morfométricas na área estudada, a filtragem realizada, mesmo não sendo a média ponderada, e mesmo os números sendo inteiros, os resultados pareceram animadores. Dessa forma, essa pode ser uma forma possível de correção do efeito dossel, mas esse tipo de procedimento não deve ser extrapolado para outras áreas com características distintas sem as devidas considerações. 


\section{Agradecimentos}

Os autores agradecem ao PGTA/UFMS (Programa de Pós-Graduação em Tecnologias Ambientais), da Universidade Federal de Mato Grosso do Sul - UFMS.

O presente trabalho foi realizado com apoio do Conselho Nacional de Desenvolvimento Científico e Tecnológico (CNPq), o qual disponibilizou a Bolsa de Produtividade em Pesquisa (Processo 305013/2018-1) de A.C. Paranhos Filho.

O presente trabalho foi realizado com apoio da Fundação Universidade Federal de Mato Grosso do Sul - UFMS/MEC - Brasil.

\section{Referências}

Abdon, M. M., Silva, J. S. V., Pott, A., Pott, V. J., Silva, M. P., Mengatto Junior, E.A. (2009). Cobertura vegetal do Parque Estadual Pantanal do Rio Negro (PEPRN) - MS. In: Simpósio De Geotecnologias No Pantanal, 2, Corumbá, 2009. Anais. São José dos Campos: Embrapa Informática Agropecuária/INPE. 674-84.

Brasil. (1988). Constituição da República Federativa do Brasil. São Paulo: Ed. Revista dos Tribunais. BRASIL.

Farr, T. G., Rosen, P. A., Caro, E., Crippen, R., Duren, R., Hensley, S., Kobrick, M., Paller, M., Rodriguez, E., Roht, L., Sseal, D., Shaffer, S., Shimada, J., Umland, J., Wener, M.; Oskin, M., Burbank, D., Alsdorf, D. (2009). The Shuttle Radar Topography Mission. Reviews of Geophysics, 45, 1-33.

Grass Development Team. (2010). Geographic Resources Analysis Support System (GRASS)

Software, Version 6.4.1. Open Source Geospatial Foundation. Recuperado de http://grass.osgeo.org.

Higa, L. T., Paranhos Filho, A. C., Torres, T. G., Salles, A. T. (2006). Avaliação da precisão vertical do modelo SRTM para a bacia do rio Paraguai, no Estado de Mato Grosso do Sul. In: $1^{\circ}$ Simpósio De Geotecnologias Do Pantanal. Campo Grande. Anais. São José dos Campos, SP:INPE. 834-840. 
Instituto Brasileiro de geografia e Estatística - IBGE. 2010. Banco de Dados Geodésicos. Recuperado de http://www.ibge.gov.br/home/geociencias/geodesia/bdgpesq_googlem aps.php\#tabela_dado).

Instituto Nacional de Pesquisas Espaciais - INPE. (2006). SPRING - Sistema de Processamento de Informações Georeferenciadas. Recuperado de http://ww w.dpi.inpe.br/spring.

Instituto nacional de Pesquisas Espaciais - INPE. (2011). Banco de Dados Geomorfométricos do Brasil. Recuperado de http://www.dsr.inpe.br/topodata/data/geotiff/.

Ludwig, R., \& Schneider, P. (2006). Validation of digital elevation models from SRTM XSAR for applications in hydrologic modeling. Journal of Photogrammetry \& Remote Sensing, $60,339-358$.

Marini, L. R., Marcato Junior, J., Ramos, A. P. M., Paranhos Filho, A. C., BarroS, W. M., Higa, L. T. (2017). Análise da acurácia Altimétrica dos Modelos Digitais de superfície SRTM, Aster e Topodata e Aplicação na Representação do Pantanal da Nhecolândia. Anuário do Instituto de Geociências, 40(3), 48 - 54.

Instituto de Meio Ambiente de Mato Grosso do Sul - IMASUL. (2008). Resumo Executivo do Plano de Manejo do Parque Estadual do Pantanal do Rio Negro. IMASUL, Campo Grande. 68 p.

QGIS Development Team. (2018). Geographic Information System. Open Source Geospatial Foundation Project. Recuperado de http://qgis.osgeo.org.

Rodriguez, E., Morris, C. S., Belz, E. A. (2006). Global Assessment of the SRTM Performance. Photogrammetric Engineering \& Remote Sensing, 72(3), 249 - 260.

Pereira, A. P., Shitsuka, D. M., Parreira, F. J., Shitsuka, R. (2018). Metodologia da pesquisa científica. Santa Maria, RS: UFSM, NTE, 119p. Recuperado de https://www.u fsm.br/app/uploads/sites/358/2019/02/Metodologia-da-Pesquisa-Cientifica_final.pdf. 
Valeriano, M. M. (2008). TOPODATA: Guia de Utilização de Dados Geomorfométricos Locais. São José dos Campos, SP. Recuperado de http://www.dsr.inpe.br/to podata/data/guia_enx.pdf.

Zyl, J. J. V. (2001). The Shuttle Radar Topography Mission (SRTM): A Breakthrough in Remote Sensing of Topography. Acta Astronautica, 48, 559 - 565.

\title{
Porcentagem de contribuição de cada autor no manuscrito
}

\author{
Alesandro Copatti - 50\% \\ Deborah Mendes - $10 \%$ \\ Ana Paula Garcia Oliveira - 10\% \\ Fabrício Bau Dalmas - 10\% \\ Antonio Conceição Paranhos Filho - 20\%
}

